\title{
Percolation of Fibrous and Bimodal Fillers in Thermoreversible Gelcast Aluminum Oxide
}

\author{
Kristina Bond
}

University of Illinois Urbana-Champaign, Department of Materials Science and Engineering

1304 W. Green St., Urbana, IL 61801

\begin{abstract}
A novel pore morphology was developed by addition of fugitive fibers to a thermoreversible gel slurry to induce pores in alumina $\left(\mathrm{Al}_{2} \mathrm{O}_{3}\right)$ samples. Through density measurements by the Archimedes' method and SEM images the percolation threshold for fibers with aspect ratios of 37 and 120 was found to be less than 1 vol\% fibers. Introducing two spherical fillers with different diameters (3.5:1 diameter ratio) to create bimodal filler has a small effect on the percolation threshold, if any. More research is needed to understand the effect of bimodal fillers on the pore morphology.
\end{abstract}

Keywords: Alumina, Gelcast, Bimodal Fillers

\section{Introduction}

A ceramic is an inorganic, brittle solid, composed of at least two elements, one of which must be a non-metal. Commonly, these ceramics are carbides, oxides, and nitrides of elemental metals or semiconductors. By nature, ceramics are strong, hard, generally non-reactive, and have very high melting temperatures. They have very low thermal and electrical conductivity ${ }^{[1]}$

These properties are useful in applications that require high strength in high-temperature and/or highly corrosive environments, such as heat exchangers, ${ }^{[2]}$ many kinds of filters,,${ }^{[3,4]}$ catalyst supports, ${ }^{[5]}$ composite preforms, ${ }^{[6]}$ and biomedical implants. ${ }^{[7-9]}$ All of these applications require a high surface area within the material, which is achieved by creating a pore network. Pores can be induced by adding organic fillers which are later removed. Other methods of pore forming include partial sintering and the addition of foaming agents. ${ }^{[10]}$

Different pore sizes and shapes are necessary for different applications. This study seeks to develop a pore morphology, or network, that is different from spherical pore morphology by introducing fibrous and bimodal filler materials at various concentrations. New network geometries will yield materials with unique mechanical, thermal, and transport properties.
Because of their very high melting temperatures, it is impractical for most applications and sometimes impossible (as in some carbides which dissociate instead of melting) to melt and cast a ceramic. Instead, solid state processing is often used. Shape forming for solid state processing can be accomplished by direct casting, in which the ceramic powder and organic binder are added to a liquid medium and mixed into slurry. The slurry is cast into a mold and is allowed to dry before proceeding to the burnout process. During burnout, the part is heated at a controlled rate which depends on the composition of the organic binders and fillers in the green body. The organics, including fillers for pore introduction, will decompose and leave only the intended pore network in the pure ceramic skeleton. The final step is to sinter the ceramic at a very high temperature, usually above $1000^{\circ} \mathrm{C}$, to complete the particle consolidation and densification of the skeleton surrounding the pores. Direct casting methods are near net-shape processes, so the final part typically does not require significant machining or finishing.

Gelcasting is a direct casting method in which the liquid and binder form a gel. Traditional gelcasting is limited because the slurry gels through an irreversible polymerization reaction, and therefore, can only be cast within a short time after the reac- 
tion catalyst has been introduced. A variant of this method, thermoreversible gelcasting (TRG), has been developed at Northwestern University. ${ }^{[11-13]}$ TRG uses the selective solubility of an acrylate tri-block copolymer dissolved in alcohol to form a gel through a physical transition instead of a chemical reaction. The tri-block copolymer has a poly(n-butyl acrylate) (PnBA) midblock and two poly(methyl-methacrylate) (PMMA) endblocks. Below the critical micelle temperature (CMT), the PMMA endblocks become insoluble and form micelles. The soluble midblocks effectively cross link the micelles, forming a gel network. The physical transition is reversible, so the gel can be reheated and recast over and over again. ${ }^{[1]}$ Thermoreversible gelcasting has additional advantages over traditional gelcasting because it is oxygen insensitive, uses safer chemicals, permits for early preparation in producing a slurry, and allows for recycling of flawed castings.

\section{Procedure}

A thermoreversible gel was made by combining 5 vol $\%$ triblock polymer PMMA $_{23}-\mathrm{PnBA}_{31}-\mathrm{PMMA}_{23}$, Kuraray Corporation, Japan) and $95 \mathrm{vol} \%$ pentanol (99+\%, Alfa Aesar) in a glass vile, then ultrasonicating the mixture in a Branson 2510 Ultrasonicator at $75^{\circ} \mathrm{C}$ until dissolved. The solution was removed from the ultrasonicator, and aluminum oxide $\left(\mathrm{Al}_{2} \mathrm{O}_{3}\right)$ with 0.05 mol\% magnesium oxide $(\mathrm{MgO})$ powder (Baikowski Malakoff, North Carolina), and the filler were added in four equal loads, each added after the previous load had completely dispersed after ultrasonication at $75^{\circ} \mathrm{C}$. Filler concentrations, in vol $\%$ of total solids, ranged from 0.5 to $5 \mathrm{vol} \%$ polypropylene (PP) or polyethylene fibers (PE) (Minifibers), or 25 to $40 \mathrm{vol} \%$ PP beadcorn starch mixture. The sizes and aspect ratios of these fillers are listed in Table I. A dispersant (Aerosol ${ }^{\circledR}$ AY 65 (sodium diamyl sulfosuccinate in ethyl alcohol) Cytec, New Jersey) was added in equal parts at the first and third solids loadings: $2 \mathrm{wt} \%$ for bimodal filler or $3 \mathrm{wt} \%$ for fiber filler.

\begin{tabular}{|c|c|c|c|}
\hline & Diameter & Length & $\begin{array}{c}\text { Aspect } \\
\text { Ratio }\end{array}$ \\
\hline $\begin{array}{c}\text { Polypropylene } \\
\text { Fiber }\end{array}$ & 30 & 1.1 & 36.7 \\
\hline $\begin{array}{c}\text { Polyethylene } \\
\text { Fiber }\end{array}$ & 5 & 0.6 & 120 \\
\hline Corn Starch & 10 & - & 1 \\
\hline $\begin{array}{c}\text { Polypropylene } \\
\text { Beads }\end{array}$ & 35 & - & 1 \\
\hline
\end{tabular}

Table I: Sizes and aspect ratios of filler materials

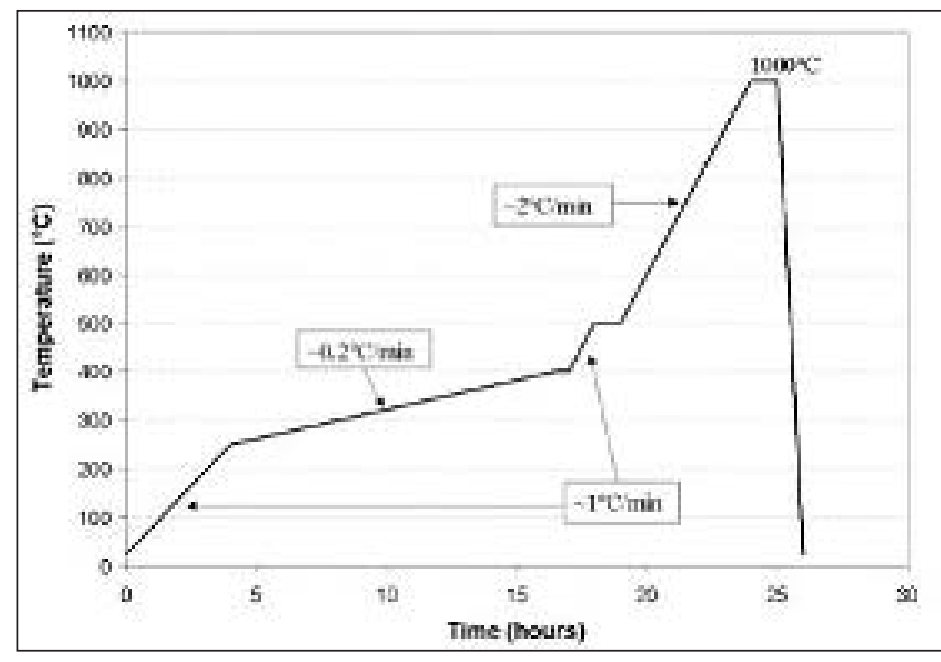

Figure 1. The burnout schedule of polypropylene fiber filler in a thermoreversible gelcast $\mathrm{Al}_{2} \mathrm{O}_{3}$

Nylon rings coated with Dow Corning Molykote 316 Silicone Release Fluid and glass slides with silicone-coated Mylar sheets (Richard E. Mistler, Pennsylvania) taped to the surface were heated on aluminum blocks in an oven at $70^{\circ} \mathrm{C}$. The glass slides were left on the aluminum blocks to keep them warm. The gel slurry was cast into the rings with a glass slide on either side, Mylar-side towards the mold. When the glass slides were cool to the touch, one slide was removed and the drying process began.

The drying process consisted of one day in a sealed, pentanol vapor environment, created by introducing a pentanol source into the sealed container. This was followed by one day in an air-tight environment (without the pentanol source), and then one day open to air.

Samples went through a burnout process that is customized to the composition of the organics. An example of the PP fiber burnout schedule is shown in Figure 1. The burnout schedules were determined from thermogravimetric analyses of the triblock copolymer and fillers. A pre-sintering step at $1000^{\circ} \mathrm{C}$ was incorporated to give the porous bodies some handling strength before the sintering process. All $\mathrm{Al} 2 \mathrm{O} 3$ samples underwent the same sintering process as follows: $10^{\circ} \mathrm{C} / \mathrm{min}$ up to $1600^{\circ} \mathrm{C}$, a one-hour hold at $1000^{\circ} \mathrm{C}$, then $10^{\circ} \mathrm{C} / \mathrm{min}$ ramp rate down to room temperature (actual ramp rate is less than $10^{\circ} \mathrm{C} / \mathrm{min}$ at lower temperatures).

The bulk density (density of the overall sample), specific gravity (density of the ceramic skeleton), and apparent porosity of samples were found using ASTM Standard C373, commonly known as the Archimedes' method. Selected samples were sectioned and polished with $0.1 \mu \mathrm{m}$ suspension to be imaged using a scanning electron microscope (Hitachi S3400), at a pressure of 70-100 Pa, using both backscattering electron (BSE) and environmental scattering electron detector (ESED) modes. 


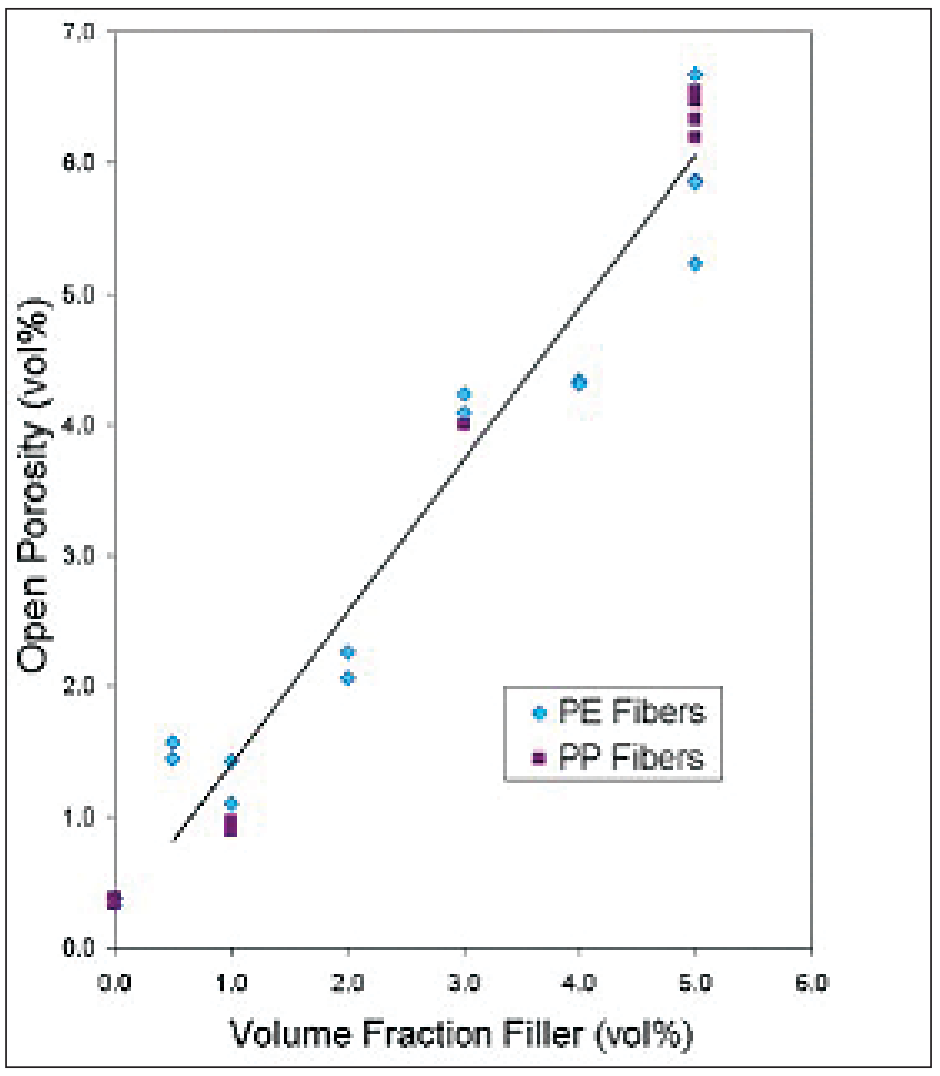

Figure 2. Open porosities of samples produced with polyethylene and polypropylene fibers as a function of volume percent filler

\section{Results and Discussion}

Pores can be classified as closed, where the individual pore or pore clusters are isolated from each other, or open, where each pore is connected to the pore network that spans the entire volume (a percolative network). A sharp boundary exists between closed and open porosity, known as the percolation threshold.

Percolation theory uses a statistical method to determine the minimum concentration of particles required to create a single, connected network in a multi-phase system. In the case of spherical, monodisperse particles in a simple cubic lattice, the percolation threshold is $31 \mathrm{vol} \%{ }^{[14]}$ The volume percent of filler material is not necessarily equal to the volume percent of the pores in the final material due to shrinkage of pores during the densification process. Percolation theory can also be useful for predicting results in non-spherical, polydisperse multi-particle systems. Since assumptions for statistical calculations and models outline ideal situations, it is necessary to evaluate pore network morphology and percolation thresholds under experimental conditions.
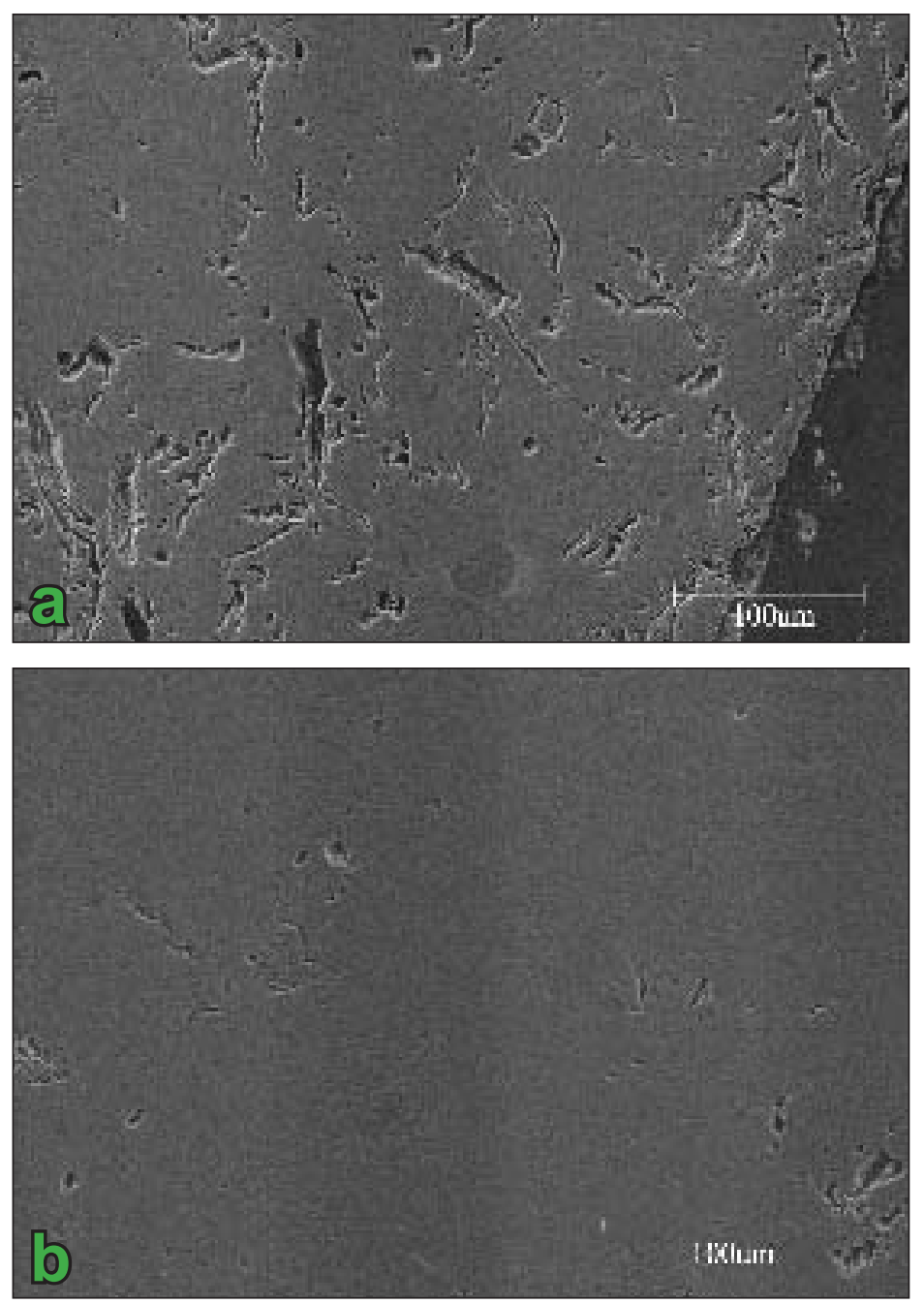

Figure 3. SEM cross-sectional images of porous $\mathrm{Al}_{2} \mathrm{O}_{3}$ produced with polyethylene fibers of random orientations at (a) 5 vol\% and (b) 1 vol\% fibers

\subsection{Fiber Fillers}

Open porosity as determined by the Archimedes' method is shown in Figure 2 for materials made with fugitive fillers of $\mathrm{PP}$ and PE fibers at concentrations between 0.5 and $5 \mathrm{vol} \%$. The apparent porosity increases linearly with volume percent fiber and porosity is proportional to filler concentration, which implies that the percolation threshold is below $1 \mathrm{vol} \%$ fugitive filler; much less than the $31 \mathrm{vol} \%$ threshold of spherical fillers. The fibers act as pre-formed channels which connect into a network at a low concentration. This is an important result because the percolation is achieved while maintaining a very high ceramics concentration, which gives a stronger structure overall.

The randomly oriented pore structures of samples produced with $5 \mathrm{vol} \%$ and $1 \mathrm{vol} \%$ PE fibers are seen in SEM images, Figure 3. The two-dimensional images of a sectioned and polished surface do not properly show the percolation of three 


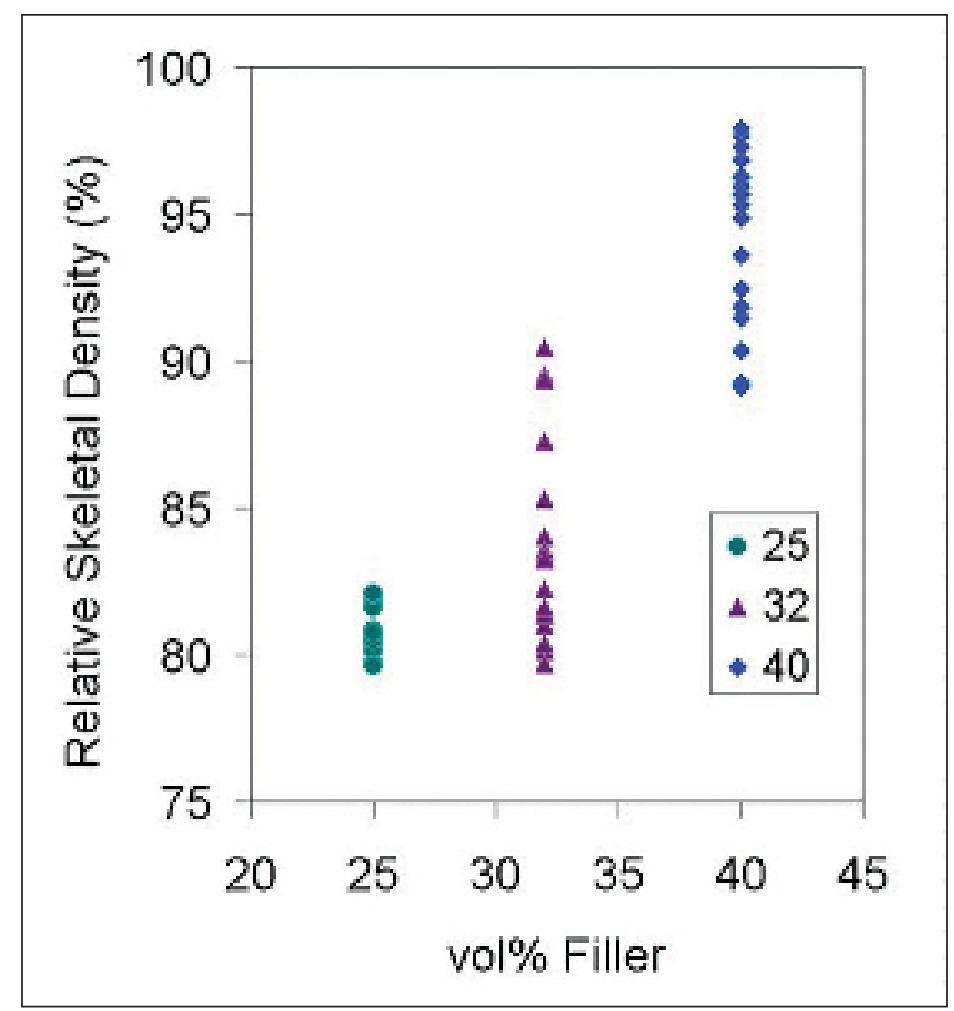

Figure 4. Relative skeletal density of samples produced with bimodal fillers of 25,32 , and 40 vol\%

dimensions. The observed plane may not display a fully-connected network even when the volume of the sample shows that, through bulk density measurements, there is complete percolation. However, the SEM images are useful to show variation of filler particle sizes and approximate ratios by the two-dimensional pore shapes, as well as to confirm random orientation of the fibers in slurry.

Modeling studies reported the percolation threshold for ellipsoids with a large range of aspect ratios. ${ }^{[15]}$ Approximating fibers as high-aspect ratio ellipsoids, the threshold for a PE fiber (aspect ratio 37) should be between 2.6 and $1.5 \mathrm{vol} \%$, and a PP fiber (aspect ratio 120) should reach percolation just below 0.69 vol $\%$. Because the cited values were generated by modeling, the numbers assume a perfect percolative system with straight particles. The flexible polymer fibers used in this study do not form perfect ellipsoids because of size dispersion and fiber bending, and the effective aspect ratios are less than modeled in the study by Garboczi, et al. ${ }^{[15]}$ Although the PP and PE fibers have different aspect ratios, results show a similar behavior for both fibers due to fiber bending which reduces the relative difference between their aspect ratios.

\subsection{Bimodal Fillers}

It was expected that the percolation threshold of bimodal fillers will deviate from $31 \mathrm{vol} \%$ based on particle packing arguments. The density and porosity of samples produced with

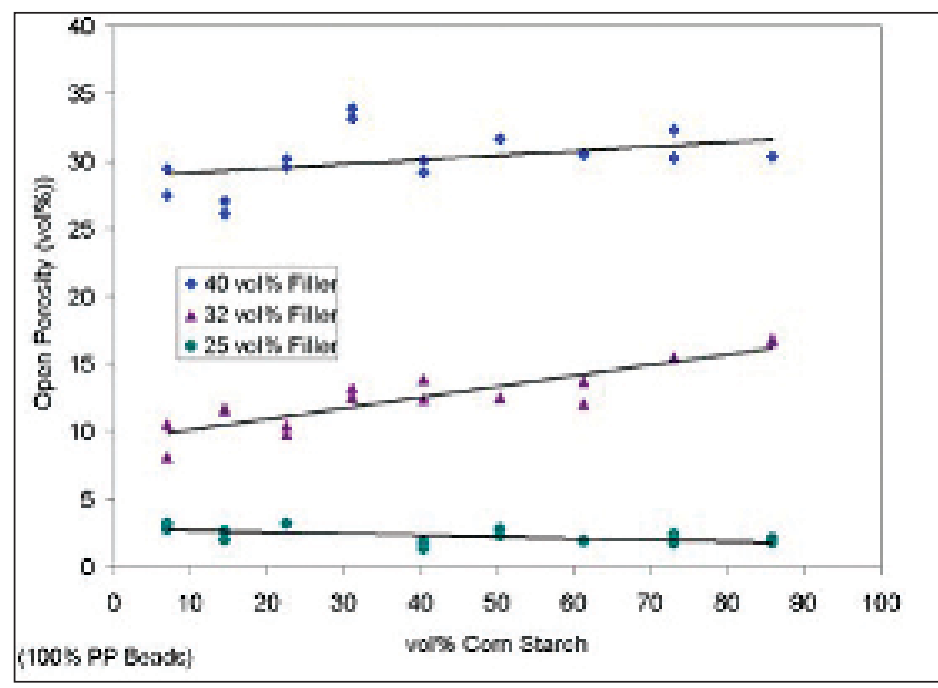

Figure 5. Open porosity as a function of cornstarch fraction for three levels of bimodal (cornstarch and polypropylene) filler

bimodal fillers at three volume percents $(25,32$, and $40 \mathrm{vol} \%)$ are plotted in Figure 4 and 5, respectively as a function of corn starch to PP bead ratio. Because Archimedes' method interprets closed porosity as a decrease in apparent specific gravity, it is expected that the percent apparent specific gravity will decrease by a percentage approximately equal to the filler concentration if closed pores are present in the sample. This is exactly the case, for samples produced with $25 \mathrm{vol} \%$ filler, where the apparent specific gravity is between 79 and $82 \%$ of theoretical density. This represents $18-21 \mathrm{vol} \%$ closed pores, which is close to the concentration of fugitive filler. More evidence that these samples do not achieve percolation is the open porosity (Figure 5), which is $\sim 3 \mathrm{vol} \%$ and less. The small amount of measured porosity could be due to surface roughness, surface cracks, or systematic error Archimedes' method, but $<3 \mathrm{vol} \%$ porosity suggests a lack of percolation in the case of bimodal spherical fillers at $25 \mathrm{vol} \%$. A SEM image of a samples produced with 25 vol $\%$ filler with 61.3 vol $\%$ corn starch is shown in Figure 6(a).

Samples with $32 \mathrm{vol} \%$ filler in Figure 4 have higher relative skeletal density than samples with $25 \mathrm{vol} \%$ filler, suggesting that there are relatively more open pores. It should be noted that corn starch has a large spread in particle size distribution, which could affect the spread of data in Figure 4. Figure 5 shows that samples produced with $32 \mathrm{vol} \%$ filler have porosities between 8 and $16 \mathrm{vol} \%$, which are too large to be surface roughness or experimental error, but too small to have reached the percolation threshold. A network has begun to form, but there are still pores in isolation from the network. Although statistical percolation is a sharp transition, the experimental system is not ideal in particle shape and size distribution; thus porosity increases gradually as the filler concentration approaches the percolation threshold. The SEM image of a sample produced 

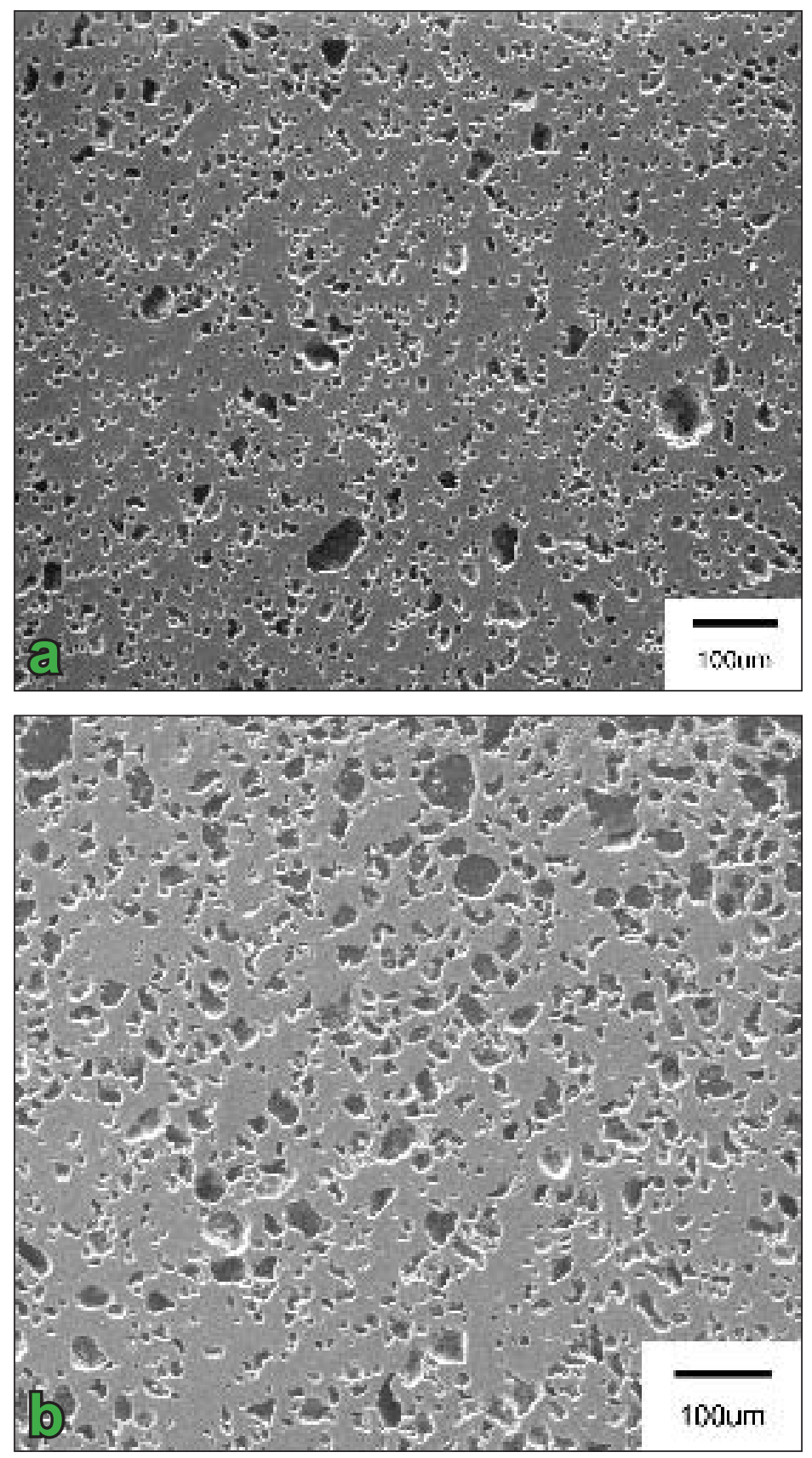

Figure 6: SEM cross sectional images of samples produced with bimodal filler at (a) 25 vol\% filler with 61.3 vol\% corn starch and (b) 32 vol\% filler with 7.0 vol\% corn starch. The percent of corn starch is the percent of total filler material.

with $32 \mathrm{vol} \%$ bimodal filler is shown in Figure 6(b).

Figure 4 shows the porosity of samples produced with 40 vol $\%$ filler to have between 89 and $98 \%$ specific gravity. The $2-11 \%$ spread from $100 \%$ density is attributed to the non-ideality of the bimodal fillers. As expected, there is evidence of percolation, although the data is not ideal. The hypothesis that the percolation threshold of bimodal fillers will deviate from 31 vol $\%$ cannot be confirmed or rejected.

\section{Conclusions}

It was found that fibrous fillers with aspect ratios of 37 and 120 reach the percolation threshold at less than $1 \mathrm{vol} \%$ filler. The results for both fibers are similar even though their aspect ratios differ by a factor of four. These results differ from the theoretically predicted result because the fibers are not ideal ellipsoids.

The percolation threshold of spherical bimodal fillers (3.5:1 diameter ratio) is between 32 and $40 \mathrm{vol} \%$ filler, but the effect of bimodal fillers as a function of particle size ratio is unclear. Any effect of bimodal fillers with size ratio 3.5:1 on percolation threshold is small due to a large particle distribution and non-ideal system. However, particle size distribution and shape appear to cause a more gradual transition from closed to open porosity instead of a sharp threshold.

\section{Acknowledgements}

Kristina would like to thank Noah O. Shanti and Dr. Katherine T. Faber for their guidance with the presented research, and the rest of the Faber group at Northwestern University for a wonderful summer in 2007. Research was funded by the National Science Foundation through the Research Experience for Undergraduates program.

\section{References}

[1] Barsoum, M W. Fundamentals of Ceramics. Institute of Physics Publishing: London, 2003; pp 2-9.

[2] Steen, M.; Ranzani, L.. Potential of $\mathrm{SiC}$ as a heat exchanger material in combined cycle plant. Ceram. Int. 2000, 26(8), 849-854.

[3] Yank, L.; Ning, X.; et al. Preparation and properties of hydroxyapatite filters for microbial filtration. Ceram. Int. 2007, 33(3), 483-489.

[4] Montanaro, L. Durability of ceramic filters in the presence of some diesel soot oxidation additives. Ceram. Int. 1999, 25(5), 437-445.

[5] Kolodziej, A; Lojewska, J. Short-channel structured reactor for catalytic combustion: Design and evaluation. Chem. Eng. Process. 2007, 46(7), 637-648.

[6] Han, J.; Hong, C.; et al. Microstructural characterization and ablation mechanism of $\mathrm{TiB} 2 /(\mathrm{Cu}, \mathrm{Ni})$ melt-infiltrated ceramic composite. Compos. Sci. Technol. 2007, 67(11-12), 2231-2237.

[7] Lacroix, D.; Prendergast, P. J.; et al. Biomechanical model to simulate tissue differentiation and bone regeneration: application to fracture healing. Med. Biol. Eng. Comput. 2002, 
40(1), 14-21.

[8] Navarro, M.; Ginebra, M. P.; et al. (2004). Development and cell response of a new biodegradable composite scaffold for guided bone regeneration. J. Mater. Sci.: Mater. Med. 2004, 15(4), 419-422.

[9] Von Doernberg, M. C., von Rechenberg, B.; et al. In vivo behavior of calcium phosphate scaffolds with four different pore sizes. Biomaterials. 2006, 27(30), 5186-5198.

[10] Reed, J. S. Principles of Ceramics Processing. pp 206

[11] Drzal, P. L; Shull, K. R. (2003). Origins of Mechanical Strength and Elasticity in Thermally Reversible, Acrylic Triblock Copolymer Gels. Macromolecules 2003, 36(6), 2002008.

[12] Montgomery, J. K., P. L. Drzal, et al. Thermoreversible Gelcasting: A Novel Ceramic Processing Technique. J. Am. Ceram. Soc. 2002, 85(5), 1164.

[13] Montgomery, J. K., Botha, A. S.; et al. A thermoreversible gelcasting technique for ceramic laminates. Scripta Mater. 2003, 48(6): 785-789.

[14] Stauffer, D. Introduction to Percolation Theory. Taylor \& Francis: Philadelphia, PA., 1985; pp 17.

[15] Garboczi, E. J., Snyder, K.A.; et al. Geometrical percolation threshold of overlapping ellipsoids. Phys. Rev. E. 1995, 52(1), 819.

\section{About the Author}

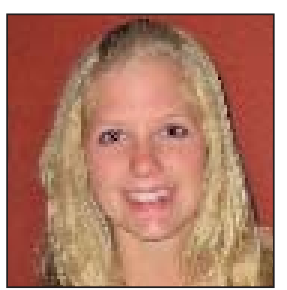

Kristina Bond is a Materials Science and Engineering student at the University of Illinois Urbana-Champaign, class of 2008. She is concentrating her undergraduate study in Biomaterials and plans to begin graduate school in biomedical engineering in the fall of 2008. Her hometown is

Shorewood, IL. 